\title{
Uma nova cultura na cultura
} institucional

\author{
Rodrigo Ramos Pinto Medeiros \\ Clara Cecchini do Prado
}

Subdividido em duas partes, o artigo relata as experiências de dois projetos um desenvolvido por Ação Educativa e outro desenvolvido pelo Centro de Estudos e Pesquisas em Educação, Cultura e Ação Comunitária (Cenpec) - que têm em comum o objetivo de propiciar a crianças e jovens que cumprem medidas socioeducativas na Fundação CASA, em São Paulo, o contato com manifestações artístico-culturais, bem como sua produção. Com vistas a alcançar esse objetivo, Ação Educativa formulou e efetivou a projeto "Arte na Casa: Oficinas Culturais”, propondo a realização de oficinas artísticas. Mais do que um projeto de acesso à cultura, a iniciativa pauta-se na produção de subjetividades individuais e coletivas por meio da ressignificação dos contextos sociais e da cultura produzida na periferia da região metropolitana de São Paulo, contexto do qual provém a maioria dos adolescentes atendidos na Fundação CASA. A segunda parte do relato traz reflexões a respeito do projeto "Educação com Arte", implementado pelo Cenpec. Nesse projeto, o fazer artístico não é entendido como ferramenta, mas como algo que tem valor em si mesmo, possibilitando a construção de um repertório cultural que confere novas formas de ver a realidade e atuar sobre ela, transformando-a.

Palavras-chave: Fundação CASA; arte-educação; crianças e jovens sob medida socioeducativas 


\title{
A new culture in institutional
}

\author{
culture \\ Rodrigo Ramos Pinto Medeiros \\ Clara Cecchini do Prado
}

Subdivided into two parts, this article reports the experiences of two projects one developed by Ação Educativa and the other developed by Centro de Estudos e Pesquisas em Educação, Cultura e Ação Comunitária (Cenpec) - that have in common the purpose of providing to children and youth taking socio-educational correctional measures at Fundação CASA, in São Paulo, the contact with artisticcultural manifestations, as well as their production. Aiming at reaching such goal, Ação Educativa formulated and carried out Project “Arte na Casa: Oficinas Culturais", proposing the realization of art workshops. More than a project for access to culture, the initiative is guided by the production of individual and collective subjectivities through the re-signification of the social contexts and culture produced in the periphery of the metropolitan region of São Paulo, a context which most adolescents served in Fundação CASA come from. The second part of the report brings reflections on the project "Educação com Arte", implemented by Cenpec. In this project, the art product is not understood as a tool, by something that has value in itself, permitting the construction of a cultural repertoire that provides new ways of seen reality and acting upon it, transforming it.

key words: Fundação CASA; art education; children and youth under socioeducational correctional measures 


\title{
Uma nova cultura na cultura institucional
}

\author{
Rodrigo Ramos Pinto Medeiros \\ Clara Cecchini do Prado*
}

Está ficando cada vez mais claro, à medida que vamos conhecendo o papel e a importância do intangível, que tudo aquilo que está ligado ao conhecimento, cultura e criatividade tem um significado, um papel no reinventar do mundo.

Lala Deheinzelin

\section{Em consonância com os direitos preconizados no} Estatuto da Criança e do Adolescente - ECA e dentro da proposta da instituição de estimular o desenvolvimento e a formação humana de jovens em conflito com a lei, a Fundação Casa vem desenvolvendo atividades de arte e cultura como parte das medidas socioeducativas.

O trabalho com arte-educação procura despertar nos jovens o olhar para as diferentes manifestações artísticas, mostrando que elas estão em todos os lugares, inclusive na periferia:

- Eles muitas vezes desconhecem esse universo [da arte]; acham que é um universo erudito, elitista, do qual não podem nem passar na frente. Não sabem que essa cultura urbana, da periferia, também é cultura" - argumenta Guilherme Astolfi Nico, gerente de Arte e Cultura da Fundação CASA. A ideia é, ao realizar essa reflexão com o jovem, dar a ele mais oportunidade de entender o mundo e assim ter mais condições de ser protagonista, agente, ator nos seus espaços.

Mas para que todo esse processo de aprendizado/ empoderamento aconteça, o papel desempenhado pelo educador é decisivo. Segundo Nico, as oficinas desenvolvidas na Fundação só adquiriram esse potencial educativo recentemente:

\footnotetext{
* Rodrigo Ramos Pinto Medeiros é sociólogo e coordenador técnico do projeto Arte na Casa: Oficinas Culturais, da ONG Ação Educativa. ClaRA CECCHINI do PRADo é atriz, pós-graduanda em Bens Culturais na FGV e foi coordenadora regional do projeto Educação com Arte: Oficinas Culturais, onde atualmente atua como especialista em Artes Cênicas.
}

- Antes, o educador chegava, definia ali o que ia fazer, muitas vezes sem um propósito a médio e longo prazos, sem uma intenção, sem saber aonde ele queria chegar com aquilo. Muitos são autodidatas e não sabiam como planejar e sistematizar aquilo que iam desenvolver.

Constatado o problema, a Fundação passou a investir na questão da formação dos educadores, dedicando a ela um dia da semana, com o propósito de discutir e trazer subsídios e respaldo teórico para os educadores, promovendo uma reflexão sobre o que é arte-educação no contexto da medida socioeducativa.

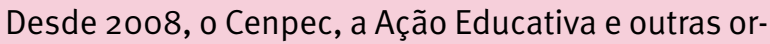
ganizações contribuem com esse esforço da instituição por meio do projeto Educação com Arte: Oficinas Culturais, que consiste na formação contínua dos arte-educadores que atuam na instituição, além da sistematização dos conhecimentos produzidos em publicações.

$\mathrm{Na}$ avaliação de Guilherme Astolfi Nico, a parceria com o Cenpec e outras instituições possibilitou um salto qualitativo no trabalho realizado pela gerência de Arte e Cultura da Fundação, reconhecido tanto pela direção da instituição como pelos próprios funcionários. Ele destaca:

- Hoje a gente percebe que as oficinas não estão sendo feitas para "tapar um buraco". Elas têm um propósito, caminham dentro da missão da Fundação, que é a de atender com qualidade esses jovens e de fato executar uma medida socioeducativa que tenha, na sua natureza, a questão pedagógica como carro-chefe e não a questão punitiva.

Nesse sentido, a continuidade e o aprimoramento das ações, bem como a divulgação dos trabalhos produzidos nas oficinas, em mostras e exposições, são fundamentais para sensibilização dos funcionários quanto ao papel da 
instituição e, consequentemente, para uma mudança da cultura interna, marcada pelo olhar preconceituoso presente na sociedade. Astolfi Nico explica:

- É esse o paradigma que nós estamos trabalhando para mudar. Mas ainda há muito que fazer, porque não é fácil mudar uma cultura instalada, enraizada em uma instituição há anos. A própria sociedade e o senso comum entendem que esses jovens são bandidos e estão aqui apenas para pagar pelo que fizeram.

Diante desse enorme desafio, ele não desanima:

- O sucesso não é efeito do acaso, mas da persistência. Acho que estamos no caminho certo."

\title{
Cultura de periferia na Fundação Casa
}

\author{
Rodrigo Ramos Pinto Medeiros *
}

Todo adolescente que cumpre medida socioeducativa de internação tem o direito a atividades de arte e cultura, estabelecido no ECA, artigos 94, inciso XI, e 124, inciso XII. Para contribuir com a garantia desse direito, entra em cena o projeto Arte na Casa: Oficinas Culturais, resultado de um convênio firmado entre a Fundação Casa e a Ação Educativa, em agosto de 2008, para atender 1216 adolescentes.

Iniciamos os trabalhos em 17 unidades e atualmente estamos presentes em 21: duas delas de internação (UI) e internação provisória (UIP); 18 de internação; e uma de internação-sanção, todas localizadas na região metropolitana de São Paulo. Esse universo nos apresenta uma gama de possibilidades de atuação, pois proporcionamos oficinas artísticas em unidades masculinas e femininas, para adolescentes com idades entre 12 e 20 anos, alguns já tendo cumprido mais de uma vez a medida socioeducativa e outros cumprindo pela primeira vez.

Isso fez com que a Ação Educativa elaborasse um projeto que contemplasse essa diversidade de atores e objetivasse principalmente a promoção da experimentação de linguagens artísticas como um exercício pedagógico e propulsor da produção de subjetividades individuais e coletivas. 0 intuito é promover a (re)descoberta de identidade e pertencimento social e o retorno dos adolescentes atendidos pela Fundação Casa à vida social.

Para atingir esse objetivo, as modalidades artísticas foram pensadas para afirmarmos uma atuação política e cultural junto aos movimentos culturais presentes nas periferias da região metropolitana de São Paulo. Dar voz a esses movimentos de maneira articulada com as atividades culturais nas unidades da Fundação Casa, junto aos adoles- centes que cumprem medidas socioeducativas, é o mote principal do projeto Arte na Casa e seu diferencial.

Acreditamos que, por meio dessa aproximação, podemos despertar no adolescente uma percepção de que seu lugar de origem não é apenas uma arena de conflitos, segregação e carências sociais. Nesse cenário de precariedade, há uma riqueza cultural da qual ele pode ser um agente ativo, altivo e promissor.

Nesse sentido, toda a nossa atuação nas unidades da Fundação Casa foi pensada para proporcionar tal aproximação. No que diz respeito às oficinas culturais, o projeto foi divido em quatro áreas artísticas, com suas respectivas modalidades. São elas: Artes do Corpo (capoeira, danças brasileiras, danças circulares e dança de rua); Artes da Palavra (literatura periférica, fanzine e rap); Artes Cênicas (teatro do oprimido, jogos dramáticos e circo); e Artes Visuais (desenho e pintura, escultura, grafite, fotografia e moda/estética). 
Pautado no Estatuto da Criança e do Adolescente, especialmente os artigos 58,71 e 121, o projeto Arte na Casa primou pelo acesso à cultura, o direito à informação, a saída do adolescente das unidades de internação e o retorno à comunidade onde sempre viveu.

Para além da promoção das oficinas artísticas, a articulação entre os movimentos culturais das periferias de São Paulo se dá de diversas maneiras. Em primeiro lugar, acontece a seleção dos arte-educadores.

Tendo em vista que diferentes estudos sinalizam que boa parte dos adolescentes privados de liberdade tem origem nas classes populares e nos bairros mais pobres da metrópole, contratamos arte-educadores pertencentes ao mesmo universo social, cultural e econômico dos adolescentes. Assim, dos 28 profissionais que ministram oficinas artísticas nas unidades da Fundação Casa, praticamente todos têm essa origem e são engajados nos movimentos culturais da periferia.

O projeto Arte na Casa: Oficinas Culturais também prevê apresentações artísticas e workshops com convidados nas unidades de internação. Essa é outra estratégia que utilizamos para levar às unidades grupos de rap, entre eles o aclamado grupo Racionais MC's, e coletivos teatrais periféricos, como o Pombas Urbanas.

Apresentamos diversos saraus com os integrantes da Cooperifa, Elo da Corrente e Sarau da Brasa. Debates com os escritores Alessandro Buzo e Sacolinha. Palestras sobre relações de gênero com o coletivo Atuadoras, questões étno-raciais com convidados e diversas outras apresentações. No primeiro ano de atividade, articulamos apresentações com quinze grupos artísticos e $34 \mathrm{ar}$ tistas que atuam nos arrabaldes da metrópole.

As formações coletivas e específicas proporcionadas aos arte-educadores pelo projeto também são meios para atingirmos nossos objetivos. Promovemos discussões sobre cultura de periferia, com agitadores culturais importantes, como Sérgio Vaz (Sarau da Cooperifa) e Alan da Rosa (Edições Toró). Planejamentos de aula focados na dinâmica de uma unidade de internação com a doutora Glória de Freitas, dentre outras palestras, reforçam nossas propostas.
Parte importante desse processo foi a valorização de outra experiência já acumulada pela Ação Educativa na divulgação e fomento da produção cultural da periferia, sobretudo com a publicação da Agenda Cultural da Periferia: um guia revelador da cena cultural de bairros mais pobres, alguns deles extremamente estigmatizados pela violência. Essa publicação passou a ser utilizada pelos educadores do Arte na Casa como um material didático que, ao apresentar a efervescência cultural periférica (tais como rodas de samba, espetáculos teatrais, saraus literários, entre outros), também contribuiu para despertar nos adolescentes privados de liberdade outra relação com suas comunidades de origem.

Os resultados dessas ações estão presentes nas pinturas, esculturas, livros de poesia, fanzines, peças teatrais, grupos de rap, grupos de dança criados pelos adolescentes. Eles, muitas vezes invisíveis e estigmatizados por grande parte da sociedade, por algum momento deixam a visibilidade e a adrenalina proporcionada pela arma de fogo, trocando-as pelo foco de luz no palco, pela ansiedade ao pegar no microfone e cantar para centenas de pessoas, pela emoção da cor e de transformar barro em obra de arte.

A literatura, muitas vezes desacreditada pelas coordenações pedagógicas das unidades, surpreendeu - possivelmente pele fato de trabalharmos com autores periféricos. Adolescentes astutos, criativos, conheceram o gosto pela leitura de maneira simples. Pegaram em livros e foram aplaudidos por suas palavras. Há relatos interessantes, como o de uma unidade da Vila Maria, na qual os adolescentes tinham vergonha de usar óculos e dois meses após a implantação da oficina de literatura a demanda foi maior que a oferta: não havia óculos suficientes para todos.

Em outra unidade, a direção implantou um sarau quinzenal com agentes de pátio, setor pedagógico e adolescentes. É o poder da palavra. 
De acordo com o pensador italiano Antonio Gramsci,

(...) o início da elaboração crítica é a consciência daquilo que somos realmente, isto é, um "conhece-te a ti mesmo" como produto do processo histórico até hoje desenvolvido, que deixou em ti uma infinidade de traços recebidos sem benefício no inventário. Deve-se fazer inicialmente este inventárioº ${ }^{1}$.
Nossa ação vai ao encontro do pensamento gramsciano, pois estamos garantindo aos adolescentes o acesso, algumas vezes uma redescoberta, aos bens culturais de suas comunidades. Eles estão privados de liberdade, porém por pouco tempo. Em breve retornarão às suas "quebradas", de volta à dura realidade. Só que agora com outros olhos, de óculos e inventário na mão.

\title{
Abrir para os jovens o universo da abstração
}

\author{
Clara Cecchini do Prado *
}

\begin{abstract}
A experiência é o que nos passa, o que nos acontece, o que nos toca. Não o que se passa, não o que acontece, ou o que toca. A cada dia se passam muitas coisas, porém, ao mesmo tempo, quase nada nos acontece. Dir-se-ia que tudo o que se passa está organizado para que nada nos aconteça ${ }^{2}$.
\end{abstract}

\section{O projeto Educação com Arte é feito de peculiaridades.}

Realizamos oficinas de arte e cultura em um ambiente em que os jovens estão separados dos seus cotidianos, exilados de suas comunidades, em um lugar em que se está de passagem. Isso gera, por exemplo, uma contínua criação e ruptura de vínculos entre adolescentes e arte-educadores e a angústia da descoberta de vocações artísticas sem possibilidade de desenvolvimento.

Além disso, trabalhar expressão e liberdade em um território que, por natureza, não pode absorver tranquilamente esse tipo de dinâmica, faz com que este projeto seja uma ação pedagógica não apenas em relação aos adolescentes, e, mesmo que de maneira indireta, atinja todos os profissionais envolvidos na execução da medida de privação de liberdade.

A contundência e a intensidade da vivência nas Unidades de Internação e Internação Provisória nos obrigam a uma reflexão constante na busca dos sentidos deste trabalho. Recusamos, porém, a pergunta "para quê?", pois está claro para todos nós que o fazer artístico e cultural é um valor em si mesmo - não usamos a medida do ferramental, não usamos "isso" para ensinar "aquilo". Preferimos a pergunta "por quê?", e sobre ela refletiremos neste texto. Para além da garantia de direitos, por que é importante que estejamos lá dentro?

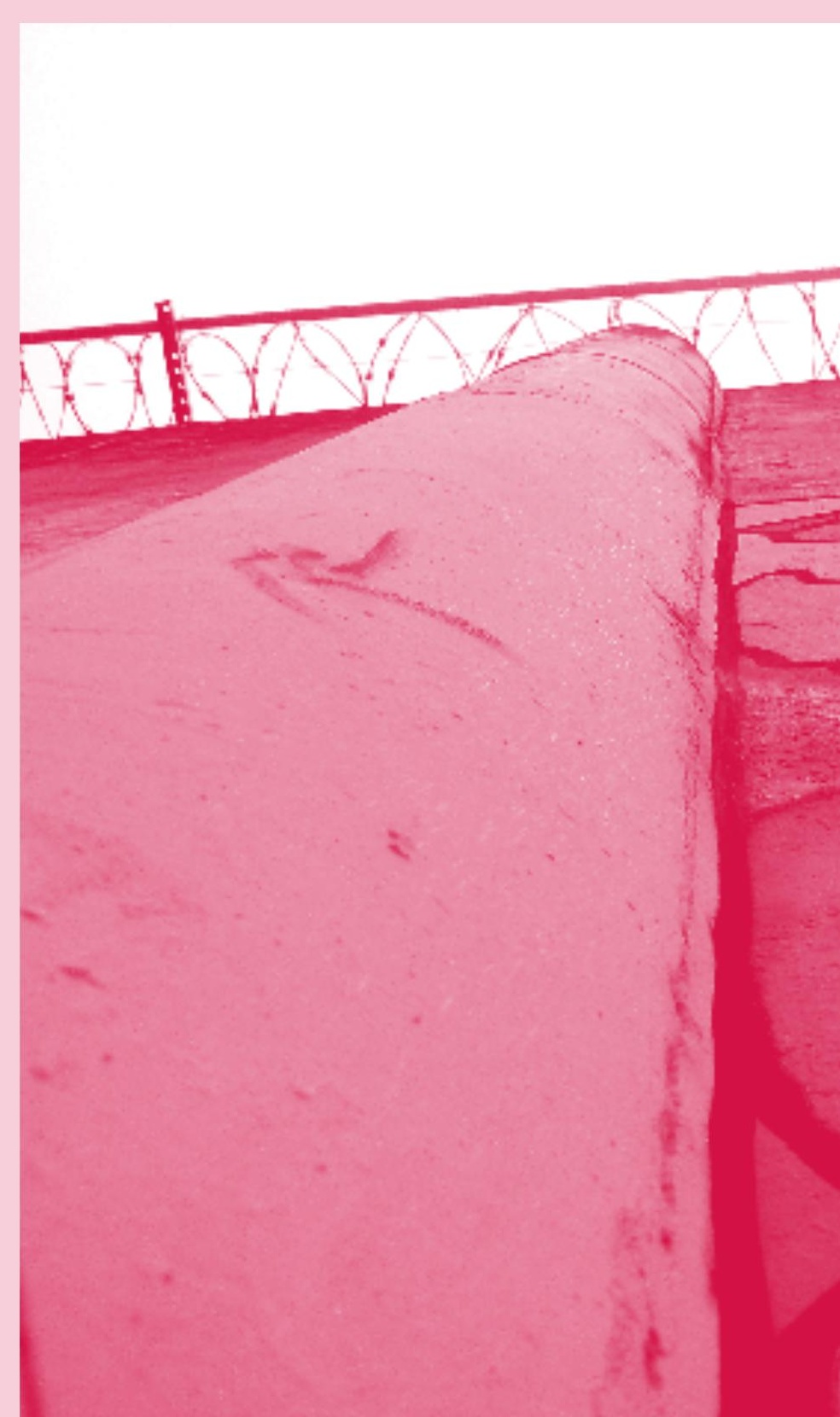


eles passarão pela medida socioeducativa.

Hoje entendemos que a experiência coletiva, compartilhada em torno de um acontecimento artístico ou cultural, é o argumento mais potente que temos para compreender o nosso papel dentro da Fundação Casa - e também para nos fazermos compreender.

Aos poucos, ganhamos cada vez mais aliados nesta empreitada que exige energia e ousadia. Pois, como é próprio da arte e da vida, experiências deste tipo contêm um alto grau de imprevisibilidade e de risco, poucas garantias, e, muitas vezes, possuem medidas que não se ajustam aos parâmetros e protocolos vigentes. Ou seja, todos os envolvidos precisam sair de sua zona de conforto - aliás, única maneira de conseguir uma transformação efetiva.

Se a própria existência cotidiana lhe parecer pobre, não a acuse. Acuse a si mesmo, diga consigo que não é bastante poeta para extrair as suas riquezas ${ }^{4}$.

\section{Notas}

1 GRAMSCl, Antonio. 1995.

2 BONDÍA, Jorge Larrosa. 2002.

3 Breakdance (também conhecido como breaking ou b-boying) é um estilo de dança de rua, parte da cultura do hip-hop, criada por afro-americanos e latinos na década de 1970 em Nova lorque, Estados Unidos. Normalmente é dançada ao som do Hip-Hop ou de Electro. $\mathrm{O}$ breakdancer, breaker, b-boy ou b-girl é o nome dado à pessoa dedicada ao breakdance e que pratica o mesmo. Inicialmente, o breakdance era utilizado como manifestação popular e alternativa de jovens para não entrar em gangues de rua que tomavam Nova lorque em meados da década de 1970. Atualmente, o breakdance é utilizado como meio de recreação ou competição no mundo inteiro. Fonte: shttp://pt.wikipedia.org/wiki/BreakdancesAcesso: 08.dezembro.2009, 21:45.

4 RILKE, Rainer Maria. 2001.

\section{Referências}

BONDÍA, Jorge Larrosa. "Notas sobre a experiência e o saber de experiência”. Conferência proferida no I Seminário Internacional de Educação de Campinas. Revista Brasileira de Educação, 끄 19. Jan/fev/mar/abr 2002. Disponível em http://www.anped.org.br/rbe/rbedigital/RBDE19/RBDE19_04_ORGE_LARROSA_BONDIA.pdf.

GRAMSCI, Antonio. Concepção Dialética da História. Rio de Janeiro: Civilização Brasileira, 1995,

RILKE, Rainer Maria. Cartas a um jovem poeta / A canção de amor e de morte do porta-estandarte Cristovão Rilke. Editora Globo, 2001.

AS FOTOS QUE ILUSTRAM ESTES RELATOS, DA PÁGINA 45 A 49, FORAM PRODUZIDAS POR ADOLESCENTES PRIVADOS DE LIBERDADE EM OFICINA CULTURAL. 